Supporting information for

\title{
Advanced electrostatic model for monovalent ions based on ab initio energy decomposition
}

Zhifeng Jing, ${ }^{\dagger}$ Chengwen Liu, ${ }^{\dagger}$ and Pengyu Ren*

Department of Biomedical Engineering, The University of Texas at Austin, Austin, TX 78712

$\dagger$ Authors that contributed equally

* Corresponding author. Email: pren@mail.utexas.edu 

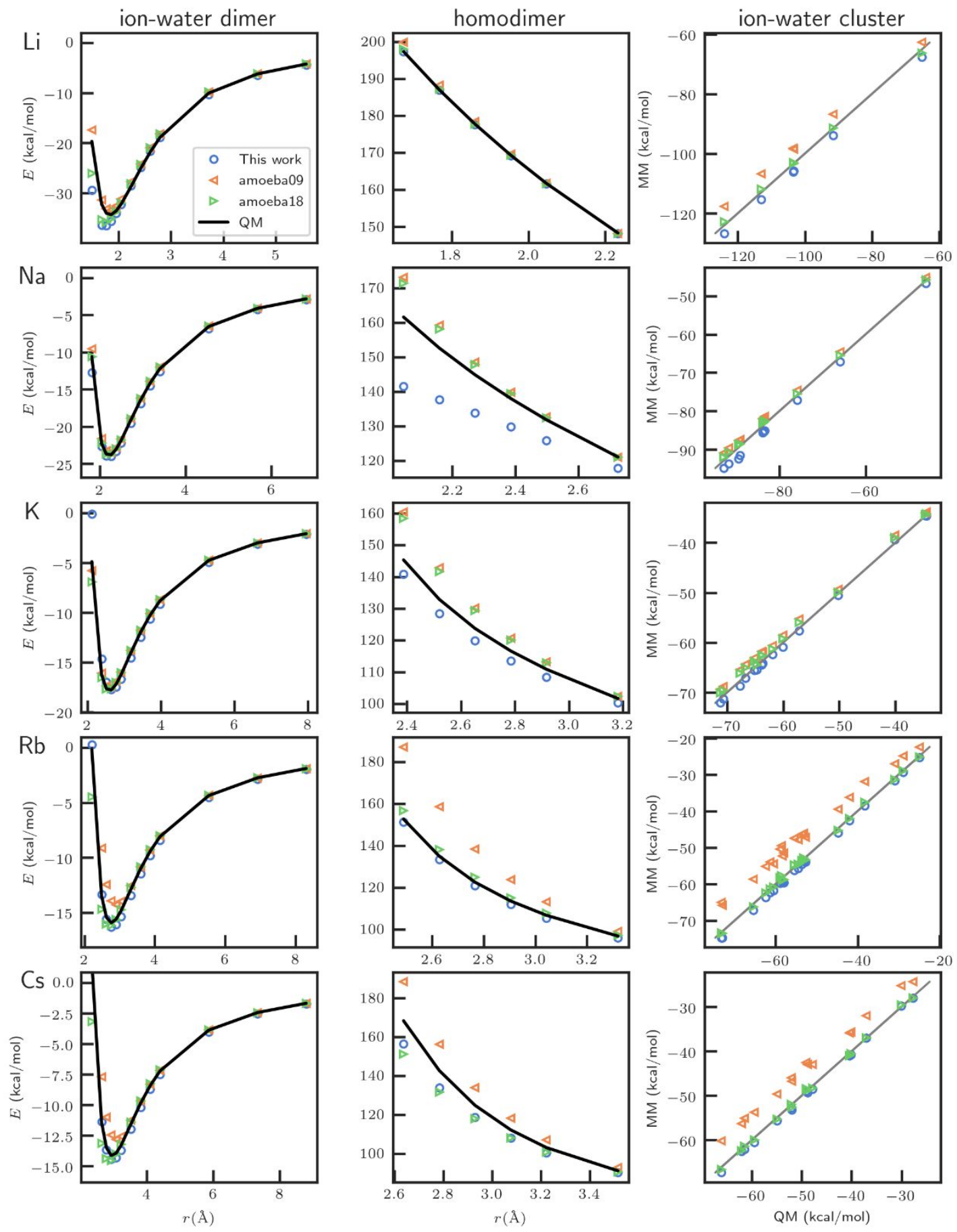

Figure S1. Comparison between AMOEBA+ and AMOEBA interaction energies for Li, Na, K, $\mathrm{Rb}$, Cs. "amoeba09" includes the $\mathrm{Na}, \mathrm{K}$ and $\mathrm{Cl}$ parameters of Grossfield et al. ${ }^{1}$ and preliminary parameters for other ions. "amoeba18" is described by Wang. ${ }^{2}$ 

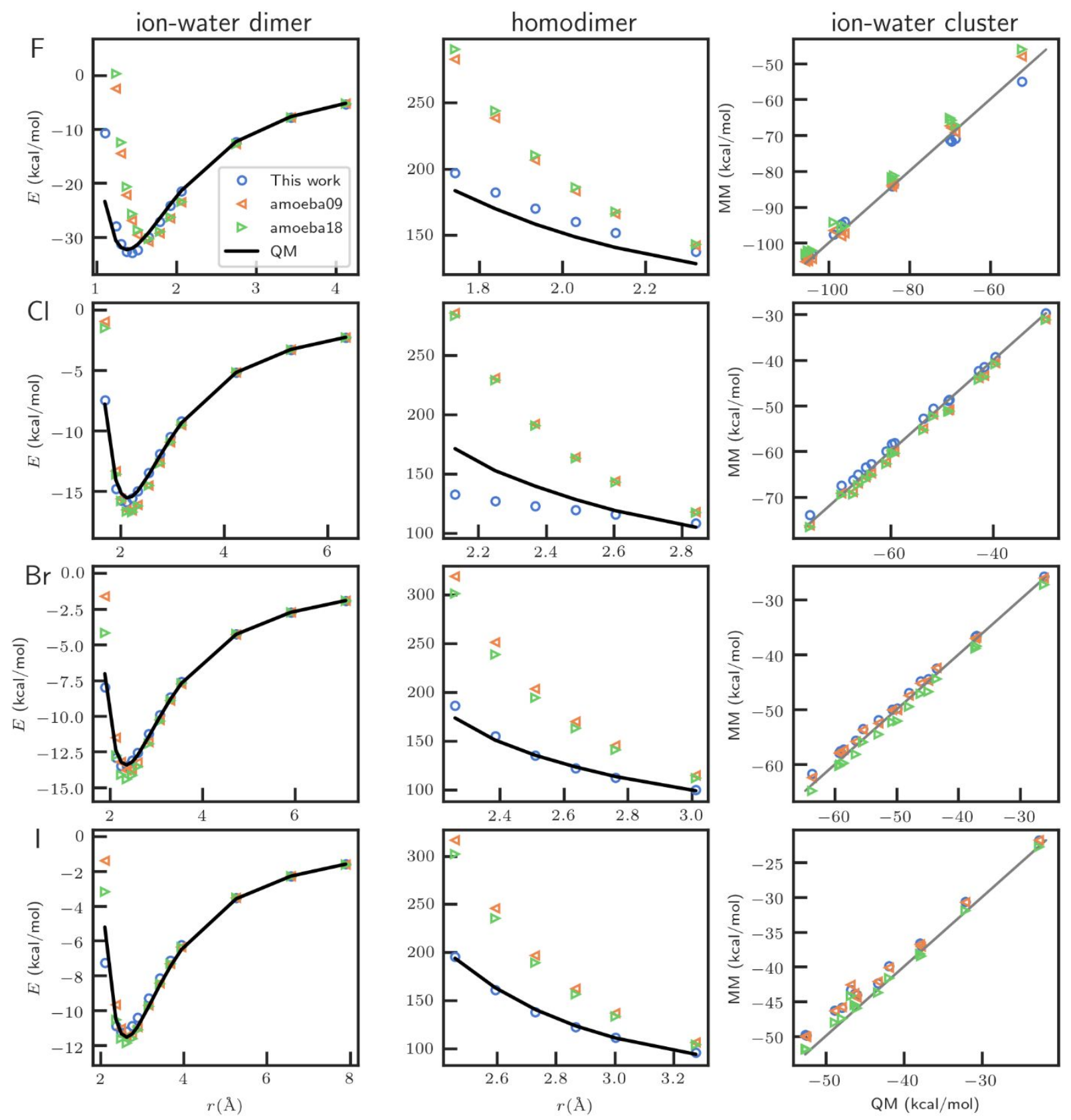

Figure S2. Comparison between AMOEBA+ and AMOEBA interaction energies for $\mathrm{F}, \mathrm{Cl}, \mathrm{Br}, \mathrm{I}$. 

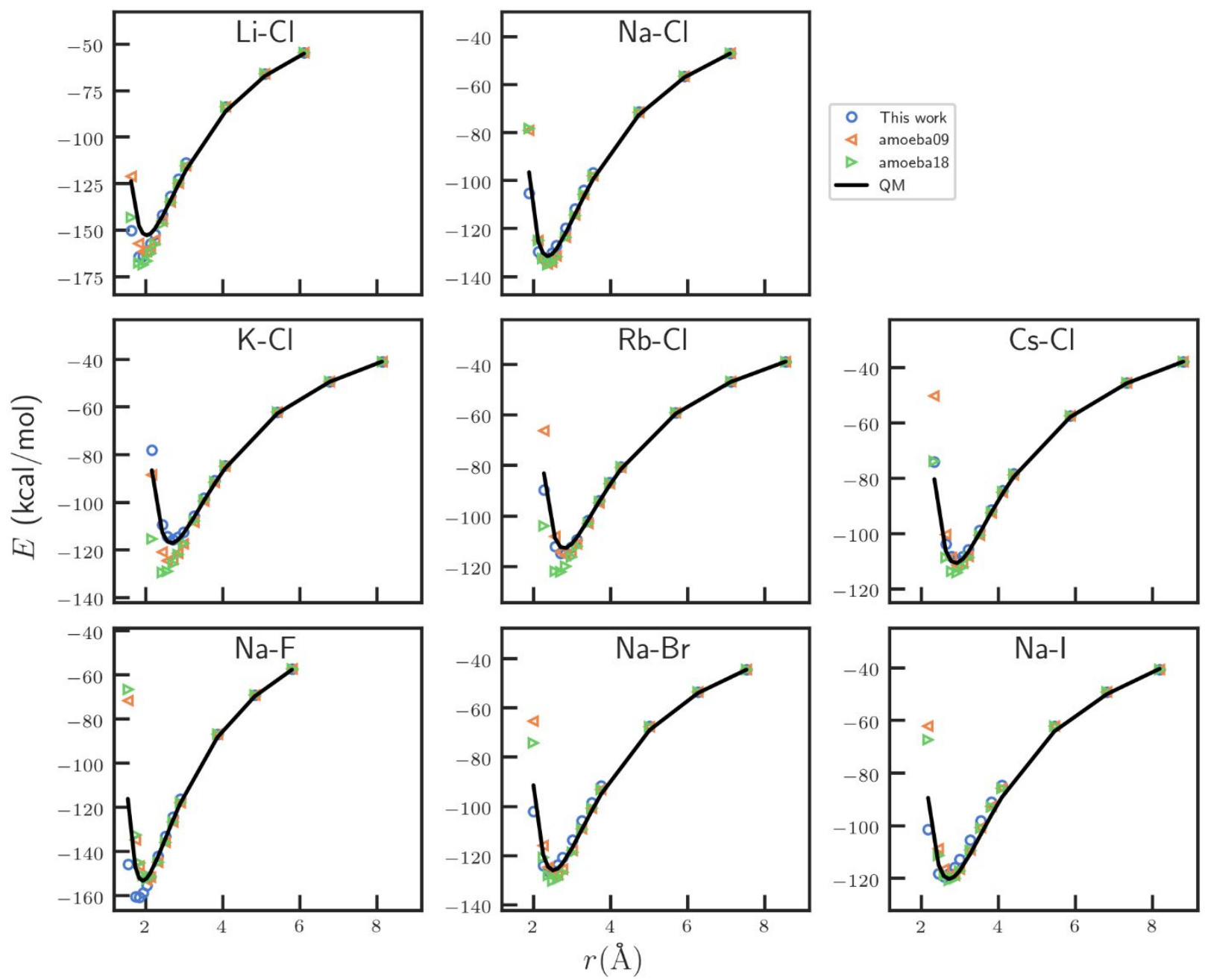

Figure S3. Comparison between AMOEBA+ and AMOEBA interaction energies for ion pairs. 


\section{REFERENCES}

1. Grossfield, A.; Ren, P.; Ponder, J. W., Ion Solvation Thermodynamics from Simulation with a Polarizable Force Field. J. Am. Chem. Soc. 2003, 125 (50), 15671-15682.

2. Wang, Z. Polarizable Force Field Development, and Applications to Conformational Sampling and Free Energy Calculation. Washington University in St. Louis, St. Louis, Missouri, 2018. 